\title{
Firmas espectrales de la cobertura de la Tierra, aplicando radiometría de campo. Fase 1: Región 03 occidente de Honduras
}

\author{
Rafael Enrique Corrales Andino \\ Vilma Lorena Ochoa López
}

\section{Resumen}

La investigación se enmarcó dentro de un proyecto nacional cuyo objetivo general es "Crear un catálogo de firmas espectrales a nivel nacional por regiones (según Plan de Nación/Visión de País) y caracterizar las coberturas de las mismas según el Sistema de Clasificación FAO, utilizando el espectro radiómetro ASD, en las bandas del espectro visible al infrarrojo cercano" para disminuir errores por dispersión atmosférica y partículas en suspensión, así como del aporte radiométrico del suelo. Se partió del hecho que el problema principal es la carencia de una base de firmas espectrales de la cobertura de la tierra, el Proyecto Nacional de Firmas espectrales tiene como Hipótesis General que: Las firmas espectrales de las diferentes coberturas de la tierra, adquiridas en campo con un espectro radiómetro, son semejantes a las firmas espectrales orbitales de la imagen corregida por dispersión de Rayleigh, siempre que la imagen sea de la misma fecha de observación. Como resultados se obtuvieron firmas espectrales de las categorías de las coberturas de la tierra: Bosque de Pino, Matorral, Agricultura, Pastos y Suelo. Para las coberturas con dominancia vegetal se aplicó el índice de vegetación de diferencia normalizada, para identificar la intensidad de biomasa con respecto a las condiciones fenológicas. Al mismo tiempo se realizó un ajuste a las condiciones del suelo, para identificar el aporte que la reflectancia de fondo (suelo) introduce en la firma espectral de la vegetación. En el análisis de la correlación entre la radiometría de la imagen corregida atmosféricamente y la radiometría de campo, realizado con las dos coberturas que presentaron mayor dificultad de separación espectral: Cafetal y Matorral, resulto que la correlación es alta, con un R2 en ambos casos de 0.99, lo que comprobó la hipótesis planteada. Y se concluyó que las firmas espectrales sirven para calibrar imágenes sin correcciones radiométricas.

Palabras clave: $\quad$ Firmas espectrales, radiometría, NDVI, SAVI, Dispersión de Rayleigh. 


\section{Abstract:}

The research formed part of a national project whose general purpose is to "Create a spectral signatures catalog nationwide, divided by region (according to National Plan / Country Vision) and characterize land cover according to the FAO classification system, using ASD FieldSpec Handheld with a visible-near infrared (VNIR) range" to reduce errors by atmospheric dispersion and particulate matter as well as ground radiometric contribution. It started from the fact that the main problem is the lack of a spectral library of land cover, the National Project of Spectral Signatures has as general hypothesis that: The spectral signatures of different land cover acquired in field with a spectroradiometer are similar to the orbital spectral signatures of the image corrected by Rayleigh scattering, as long as the image has been acquired at the same observation date. As results, Pine Forest, Thicket, Agriculture, Pasture and Soil spectral signatures were obtained. For dominant vegetable toppings Normalized Difference Vegetation Index was applied to identify the intensity of biomass regarding phenological conditions. At the same time an adjustment to soil conditions was performed to identify the contribution of background reflectance (ground) introduced into the spectral signature of vegetation. In the analysis of the correlation between radiometric measures for image corrected atmospherically and field spectroradiometry, perform with both coverage that provided the most difficult separation, cafetal and thicket, they provided a high correlation coefficient, with a R2 of 0.99 in both cases, which proved the hypothesis. The study concluded that spectral signatures are useful to calibrate images without radiometric corrections.

Keywords: spectral signatures, radiometry, NDVI, SAVI, Rayleigh scattering.

Rafael Enrique Corrales Andino (rcorrales@unah.edu.hn), Vilma Lorena Ochoa López (vl_ ochoa@yahoo.com.mx).Departamento de Ciencia y Tecnologías de la Información Geográfica, Facultad de Ciencias Espaciales, Universidad Nacional Autónoma de Honduras 


\section{Introducción}

La aplicación de las diferentes técnicas de Teledetección, cada vez más demanda que se trabaje con bases de datos con atributos radiométricos de las diversas coberturas de la tierra y del uso que a estas se le esté dando. En este sentido a continuación se describirán algunas de las técnicas y herramientas que se suelen utilizar para realizar proyectos de teledetección aplicada.

Para tener una visión directa de las características del terreno, es necesario obtener mediciones radiométricas de reflectividad, o firmas espectrales de las distintas coberturas. Esto es muy útil para que el intérprete entienda la información de los datos de Teledetección y el terreno. Como este tipo de datos puede estar afectado por condiciones atmosféricas, se trata de generar la información en la fecha y hora en que el sensor de teledetección captura los datos, también para reducir errores, por la escala de las imágenes en relación con la complejidad del terreno y la diferencia de tiempo entre el trabajo de campo y las fechas de adquisición de las imágenes satelitales.

Honduras no cuenta con una base de datos nacional de firmas espectrales de las diferentes coberturas de la tierra, que permita validar proyectos de teledetección (Percepción Remota). La gran mayoría de los estudios realizados en el país con técnicas y análisis de imágenes satelitales, solo cuentan con radiometría orbital, lo que en muchos casos dificulta la precisión en la clasificación de las coberturas por la influencia negativa de los parámetros atmosféricos y prácticas agrícolas como la quema de bosques, guamiles y pastos, provocando una identificación difusa y aumentando la dispersión de la reflectividad, principal causante de errores y excesiva edición de imágenes satelitales, específicamente de clasificación de coberturas y dinámica de cambios en la cobertura.

Contando con un primer producto de clasificación de coberturas de la tierra y de estudios radiométricos de un sector del Occidente de Honduras, publicado por Pineda de Carías, Ochoa y Corrales (2012), este nos sirvió de punto de partida para iniciar un estudio de firmas espectrales de la cobertura de la tierra, del cual se deriva un catálogo de firmas espectrales.

Entre las muchas aplicaciones de generar un catálogo de firmas espectrales de diversas coberturas del suelo, se citan las más comunes en el ámbito técnico científico (Gerrit F. Epema, 1999), como siguen: 
- Establecer relaciones entre reflectividad y características de la cobertura.

- Calcular las correlaciones entre la reflectividad medida sobre el terreno y la obtenida a partir de los niveles digitales (ND). Con lo que podemos calibrar sin correcciones atmosféricas.

- Analizar datos multitemporales. Si se conoce la reflectividad sobre el terreno de diferentes lugares en distintas fechas se puede determinar en qué medida un cambio en la reflectividad puede deberse a un cambio en la propia superficie 0 en los factores externos como la atmósfera o el ángulo cenital solar.

- Revelar las causas de una reflectividad específica en pixeles homogéneos 0 heterogéneos. Esto nos permitirá realizar una interpretación más fiable de los tonos de gris y colores de la imagen.

- Calibrar los resultados de modelos basados en valores de reflectividad.

- Detectar la influencia de cambios naturales o artificiales en la cubierta. Por ejemplo cubiertas con alto contenido de humedad o secas. Esta información puede ser muy útil para analizar imágenes de satélite $u$ otro tipo de datos obtenidos a partir de la teledetección que presenten grandes diferencias en cuanto a su contenido en humedad.

- Comprobar modelos de reflectividad, por efecto de la dirección del nadir o por el efecto BRDF (Bidirectional Reflectance Distribution Function).

- Examinar la influencia de factores externos: en diferentes condiciones atmosféricas y con diferentes ángulos cenitales solares.

En diferentes proyectos de investigación que se han venido desarrollando en la Facultad de Ciencias Espaciales (FACES), a través del Departamento de Ciencia y Tecnologías de la Información Geográfica (DCTIG) y la Maestría en Ordenamiento y Gestión del Territorio (MOGT), se utilizan imágenes satelitales principalmente para realizar análisis de cobertura y uso del suelo y análisis multitemporales, por lo que el catálogo de firmas espectrales a nivel nacional, es una contribución importante para el desarrollo de las investigaciones en el campo de la percepción remota (teledetección) aplicada. Además, la validación del protocolo de trabajo desarrollado por Cáceres (2013), para el Laboratorio de Radiometría del DCTIG de la FACES-UNAH, fue otro aporte importante de esta investigación. 


\section{Marco referencial}

En Honduras el campo de la Teledetección se venía desarrollando lentamente, pero no fue hasta después del paso del Huracán Mitch que esta tecnología tomó mayor importancia, particularmente en instituciones de gobierno o de cooperación internacional relacionadas con el manejo de los Recursos Naturales. En instituciones como la Secretaria de Recursos Naturales y Ambiente, el Instituto de Conservación Forestal, la Cooperación Alemana Internacional GIZ (por sus siglas en alemán), se han desarrollado proyectos orientados principalmente al análisis de imágenes satelitales para estudiar la cobertura de la tierra y su dinámica de cambio, la gestión del riesgo, y el ordenamiento territorial y la vulnerabilidad (INTELSIG, 2003).

En la FACES, se han desarrollado proyectos de investigación en el campo de la teledetección aplicada, orientados principalmente al análisis de cambio de la cobertura de la tierra, detección de anomalías hidrotermales y al estudio sitos arqueológicos (Corrales, et. al. 2004; Corrales, 2009; Ochoa, 2005; Sánchez Ramos, 2009; Pineda de Carías et. al., 2012; Rodríguez, 2014). Corrales en 2009 y 2010, trabajo en la metodología de captura de reflectancia de diferentes masas vegetales y en el primer catálogo de firmas espectrales de la ciudad universitaria, utilizando el espectro radiómetro JEULIN Radiameter 545031, que trabaja con seis bandas del espectro electromagnético $(0.4-1.1 \mu \mathrm{m})$.

En el año 2010, en el marco del I Congreso Internacional de Ordenamiento Territorial y Tecnologías de la Información Geográfica, realizado en la Ciudad Universitaria/UNAH, se desarrolló el Taller "Sensores Remotos y Radiometría de Campo", en el cual los participantes fueron capacitados en el uso del espectro radiómetro ASD (Martín y Sánchez, 2010 en faces.unah.edu.hn/ctig/sitios/congreso/ programdetal.html).

\section{Marco contextual}

La unidad de análisis fue la Región 03 Occidente de Honduras. La Población: lo constituyeron las diferentes Coberturas de la Tierra en la región (Figura 1). $Y$ la Muestra, fue seleccionada en base a los siguientes criterios de prioridad de análisis (PA):

Costo de interpretación espectral, el cual está relacionado con la separabilidad en la clasificación espectral de las diferentes coberturas y usos. Clasificando el nivel en costo bajo, medio y alto. 
Presión antrópica, relacionado con la presencia e intensidad de actividades humanas en las coberturas clasificadas, como presión baja, media y alta.

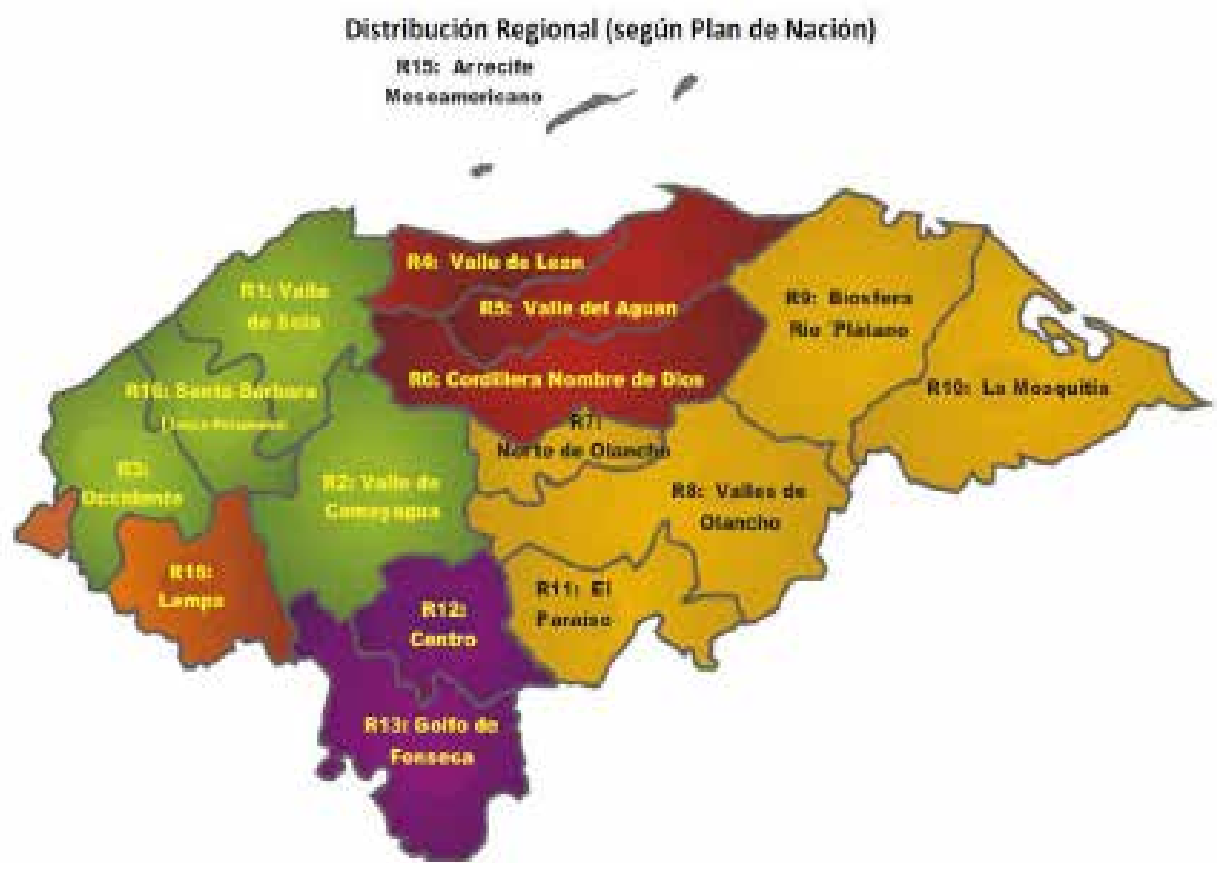

Figura 1. Distribución de Regiones de Desarrollo de Honduras.

A cada criterio se les asignan pesos (W) para calcular el valor ponderado (VP), que determinara la prioridad de análisis de cada cobertura (cuadro 1).

\begin{tabular}{|l|l|l|l|l|l|l|l|}
\hline \multirow{3}{*}{ Clases } & \multicolumn{3}{c|}{$\begin{array}{c}\text { Costo de Interpretación } \\
\text { Espectral }\end{array}$} & \multicolumn{3}{c|}{ Presión antrópica } & \multirow{2}{*}{ VP } \\
\cline { 2 - 9 } & Bajo & Medio & Alto & Bajo & Medio & Alto & \\
\hline Bosque Mixto & 1 & & & 1 & & & 1 \\
\hline Bosque Pinar & & 2 & & & 2 & & 4 \\
\hline Matorral & & & 3 & & & 3 & 9 \\
\hline Pastos & & 2 & & & & 3 & 6 \\
\hline Agricultura & & 2 & & & & 3 & 6 \\
\hline Urbano & 1 & & & 1 & & & 1 \\
\hline
\end{tabular}

Cuadro 1. Matriz de prioridad de análisis (PA) de cobertura y uso de la tierra.

Las variables investigadas fueron cobertura y radiometría, siendo la radiometría la variable dependiente, y el comportamiento de la firma espectral se ve afectado por las características de la cobertura. La variable cobertura presenta dos 
dimensiones de análisis, que son cobertura de la tierra y uso del suelo. Y la variable radiometría, sus dimensiones son radiometría de campo y radiometría orbital.

\section{Metodología}

La investigación busco demostrar relaciones entre parámetros biofísicos y la información espectral de la imagen satelital. La metodología seguida en la investigación comprendió cinco etapas (ver Figura 2): 1) Revisión bibliográfica, 2) Identificación de coberturas por Clasificación FAO, 3) Gira de campo y utilización de Radiómetro ASD, Recolección y almacenamiento de los datos de Reflectancia, 4) Construcción de fichas para el Catálogo de Firmas Espectrales Web, y 5) Publicación de los Datos.

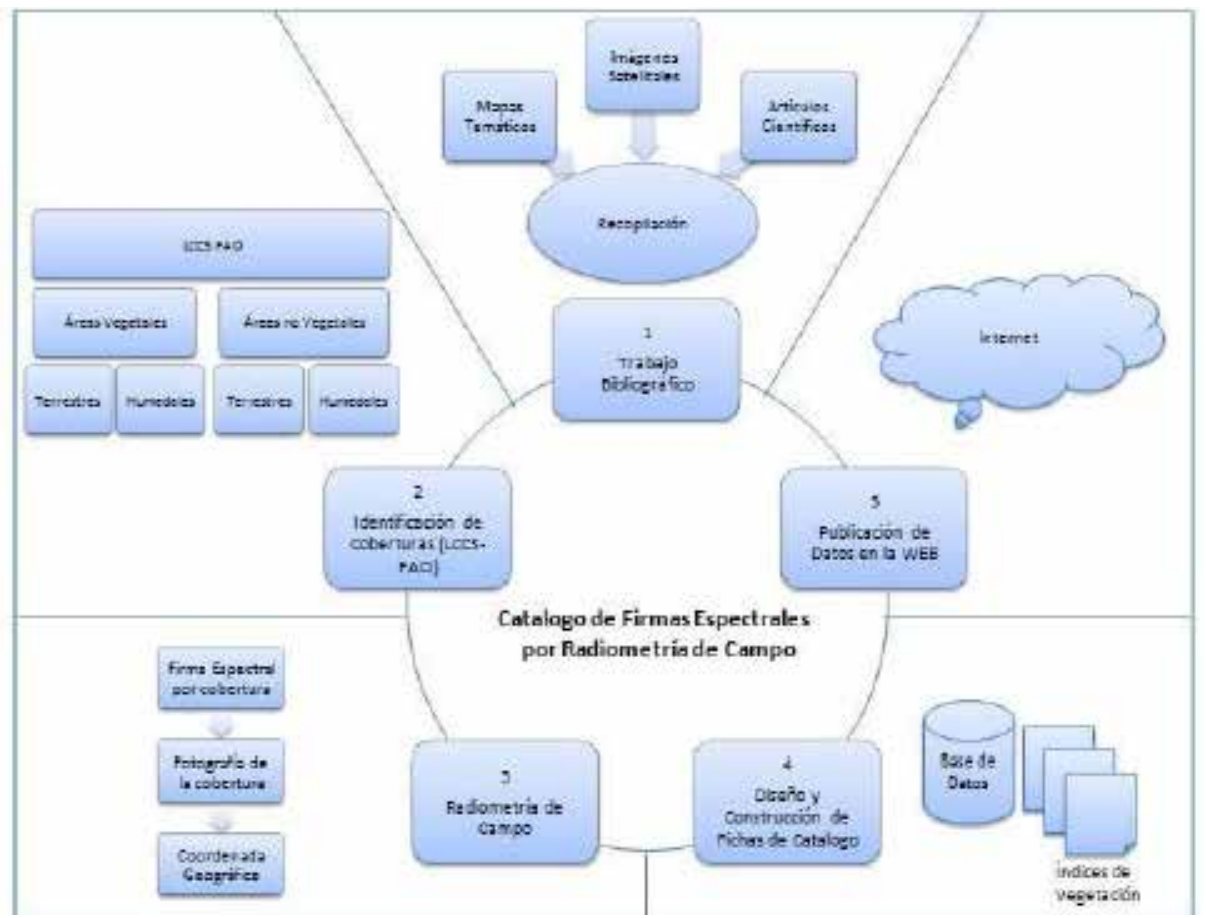

Figura 2. Diseño metodológico de la investigación. Elaboración propia. 


\section{Resultados}

En la investigación se seleccionaron y analizaron diez coberturas: i) área quemada, ii) cafetal, iii) cultivo de piña, iv) pasto verde, v) pasto seco, vi) matorral, vii) pino joven, viii) suelo desnudo con escasa vegetación, ix) suelo claro y $\mathrm{x}$ ) suelo rojo. Para cada cobertura se analizó la reflectancia de acuerdo a las bandas del sensor y se calculó el Índice de Vegetación de Diferencia Normalizada (NDVI) y el Índice de Vegetación Ajustada al Suelo (SAVI).

1. Validación de Protocolo de radiometría de campo.

Los resultados obtenidos al aplicar la Guía Rápida para captura de radiometría ASD FieldSpec HandHeld Pro (ASD Inc., 2003), que define nueve pasos para captura y registro de firmas espectrales, por tanto la aplicación de la guía fue efectiva y permitió el registro de las firmas correspondientes para cada cobertura seleccionada.

2. Identificación de las coberturas de la Región 03, a través del Sistema de Clasificación LCCS FAO-UNEP, para encontrar su firma espectral.

La identificación y selección de las coberturas del área de estudio, se basó en los resultados alcanzados en la investigación realizada por Pineda de Carías, Ochoa y Corrales (2012), donde la cobertura se clasifico de acuerdo al sistema clasificación de Cobertura de la Tierra de la FAO-UNEP (cuadro 2). 


\begin{tabular}{|c|c|c|c|}
\hline Coberturas LCLU & Clases FAO & Características & Concentración \\
\hline Bosque Mixto & Clase Mixta & $\begin{array}{l}\text { Arboles altos de hoja ancha, } \\
\text { fragmentada, con árboles altos de } \\
\text { hoja acicular dispersos }\end{array}$ & {$[(20-10)-4 \%]$} \\
\hline Bosque de Pino & Bosque & Arboleda de hoja acicular (Pino) & $65-15 \%$ \\
\hline Matorral & Arbusto & $\begin{array}{l}\text { Arbustos de hoja ancha de } \\
\text { medianos a altos }\end{array}$ & $65-15 \%$ \\
\hline Pastos & Clase Mixta & $\begin{array}{l}\text { Cultivo cambiante de herbáceas } \\
\text { cosechadas }\end{array}$ & continua \\
\hline Agricultura & Clase Mixta & $\begin{array}{l}\text { Área permanentemente cultivada } \\
\text { con arbustos cultivados con agua } \\
\text { de lluvia, coberturas cultivadas en } \\
\text { huerto, cobertura cultivada en } \\
\text { plantación, cultivos cambiantes de } \\
\text { no gramíneas, con cultivos de } \\
\text { herbáceas. }\end{array}$ & simultánea \\
\hline Urbano & $\begin{array}{l}\text { Áreas } \\
\text { construidas }\end{array}$ & Áreas urbanas & No lineal \\
\hline Suelo Desnudo & Suelo desnudo & $\begin{array}{l}\text { Áreas desnudas no consolidadas. } \\
\text { Suelo desnudo pedregoso y/u } \\
\text { otros(s) material(es) no } \\
\text { consolidado(s) }\end{array}$ & $\begin{array}{l}40-5 \% \\
\text { pedregosidad }\end{array}$ \\
\hline
\end{tabular}

Cuadro 2. Cobertura de la Región 03, a través del Sistema de Clasificación LCCS FAO-UNEP.

$Y$ en el área de estudio se identificaron cinco clases de acuerdo al sistema de clasificación FAO-UNEP, algunas de ellas por las características que presentaban, fueron desagregaron en sub clases:

- Bosque de Pino: Para fines de esta investigación se clasifico como Pino joven, debido a la predominancia de árboles inmaduros.

- Matorral: En esta cobertura predominaban arbustos de hoja ancha, de mediana altura y hierbas leñosas.

- Agricultura: En cobertura se identificaron dos Sub-clases, Cultivo de Piña y Cafetal

- Pastos: En base a las condiciones fenológicas de esta cobertura se identificaron dos sub-clases Pasto Seco y Pasto Verde. 
- Suelo: Esta clase fue subdividida en tres sub-clases, en función de la coloración del suelo y la vegetación, resultando las sub-clases: Suelos rojos, Suelos Claros y Suelo desnudo con escasa vegetación.

Además, es idéntico una sexta categoría, que se denominó área quemada.

3. Establecimiento las Fichas Digitales de las firmas espectrales de la Región 03 Occidental de Honduras, para su publicación WEB, en la página de la unidad académica del Departamento de Ciencia y Tecnologías de la Información Geográfica de la Facultad de Ciencias Espaciales de la UNAH.

A partir de las firmas espectrales obtenidas mediante la radiometría de campo, para cada una de las 10 clases de cobertura de la tierra, se elaboró la correspondiente Ficha Digital (Figura 3), que contiene la siguiente información:

- Nombre de la cobertura

- Descripción de la cobertura

- Interpretación de la firma espectral

- Coordenadas geográficas

- Fecha y hora de adquisición de la firma

- Código de la firma

- Fotografía de la cobertura.

- Calcular correlaciones entre radiometría orbital a tope de atmósfera, radiometría por corrección atmosférica de Rayleigh y radiometría de campo.

Los resultados de esta investigación se presentan discretizados por las cuatro bandas espectrales del sensor Landsat ETM+ (radiometría orbital), que son las primeras cuatro bandas que realmente registran el comportamiento espectral y que corresponden al dominio del rango visible y del infrarrojo cercano (cuadro 3). 

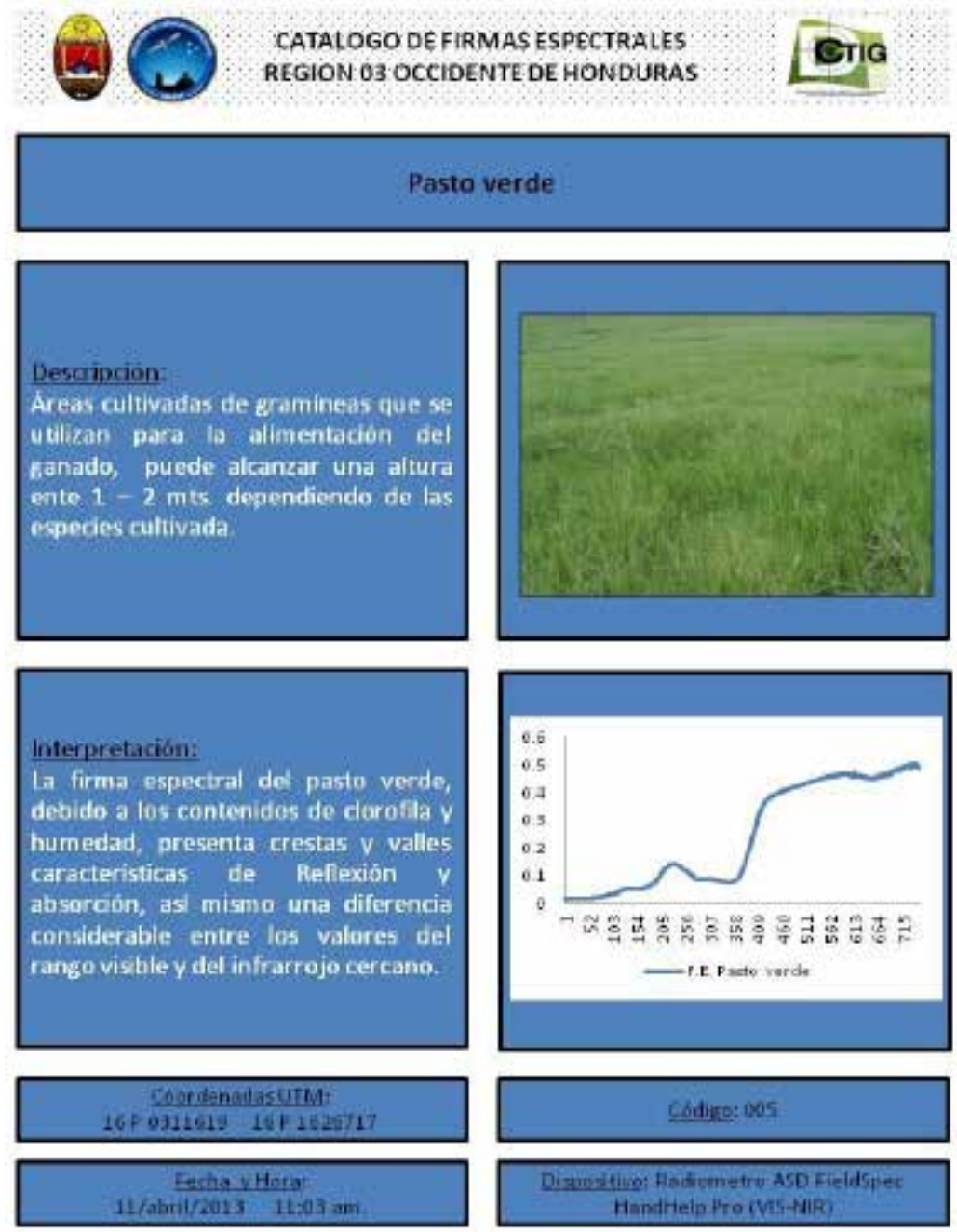

Figura 3. Ficha Digital del Catálogo de firmas espectrales correspondiente a pasto verde, en la cual notamos la imagen de la cobertura y sus características espectrales y espaciales. 


\begin{tabular}{|c|c|c|c|}
\hline Banda & Rango Espectral & Centro de Banda & Nombre de la Banda \\
\hline \multirow{2}{*}{1} & $0.450-0.515$ & 0,485 & Azul \\
\cline { 2 - 4 } 2 & $0.525-0.605$ & 0,57 & Verde \\
\cline { 2 - 4 } 3 & $0.630-0.690$ & 0,66 & Rojo \\
\hline \multirow{2}{*}{4} & $0.780-0.900$ & 0,84 & Infrarrojo cercano \\
\cline { 2 - 4 } 5 & $1.550-1.750$ & 1,65 & Infrarrojo medio \\
\cline { 2 - 4 } 6 & $2.090-2.350$ & 2,22 & Infrarrojo medio \\
\hline \hline
\end{tabular}

Cuadro 3. Rango espectral de las bandas del sensor LandSat ETM+.

Fuente: Modificado de Fernández Copel y Herrero Llorente, (s/a.)

La imagen utilizada para la extracción de firmas espectrales orbitales fue Landsat ETM+ escena P019R050, correspondiente a la región de cubre el sector Occidente de Honduras.

En el cuadro 4 se registra la reflectancia de las 10 coberturas seleccionadas, a partir de esta información se muestran las firmas espectrales de las coberturas: i) Firmas a partir de las 6 bandas del sensor (figura 4) y ii) Las firmas de las bandas del rango visible y el infrarrojo cercano (figura 5).

\begin{tabular}{|l|c|c|c|c|c|c|}
\hline \multirow{2}{*}{ Coberturas } & \multicolumn{7}{|c|}{ Bandas Espectrales } \\
\cline { 2 - 7 } & $\mathbf{0 , 4 8 5}$ & $\mathbf{0 , 5 7}$ & $\mathbf{0 , 6 6}$ & $\mathbf{0 , 8 4}$ & $\mathbf{1 , 6 5}$ & $\mathbf{2 , 2 2}$ \\
\hline Área quemada & 0,05 & 0,07 & 0,025 & 0,06 & 0,19 & 0,18 \\
\hline Suelo desnudo & 0,06 & 0,07 & 0,06 & 0,2 & 0,2 & 0,09 \\
\hline Cultivo de piña & 0,07 & 0,08 & 0,09 & 0,15 & 0,28 & 0,17 \\
\hline Pasto Seco & 0,06 & 0,07 & 0,08 & 0,15 & 0,2 & 0,12 \\
\hline Pasto Verde & 0,06 & 0,06 & 0,07 & 0,14 & 0,24 & 0,12 \\
\hline Matorral & 0,06 & 0,06 & 0,06 & 0,16 & 0,15 & 0,08 \\
\hline Cafetal & 0,17 & 0,22 & 0,26 & 0,22 & 0,34 & 0,24 \\
\hline Suelo claro & 0,07 & 0,08 & 0,09 & 0,14 & 0,33 & 0,21 \\
\hline Pino joven & 0,06 & 0,08 & 0,08 & 0,14 & 0,31 & 0,18 \\
\hline Suelo rojo & 0,06 & 0,08 & 0,08 & 0,18 & 0,23 & 0,12 \\
\hline
\end{tabular}

Cuadro 4. Radiometría de Imagen Landsat ETM+, para 10 coberturas 


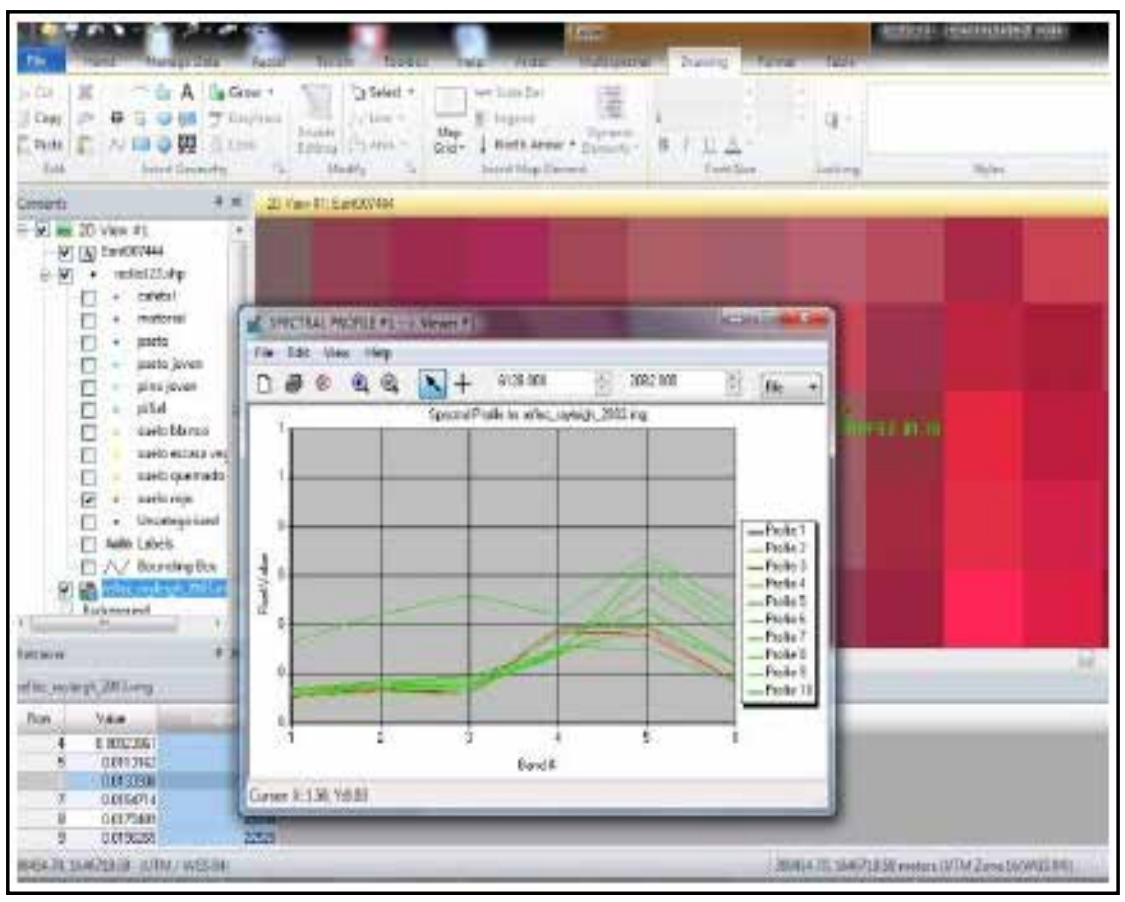

Figura 4. Extracción de firmas espectrales de la imagen del satélite LandSat 


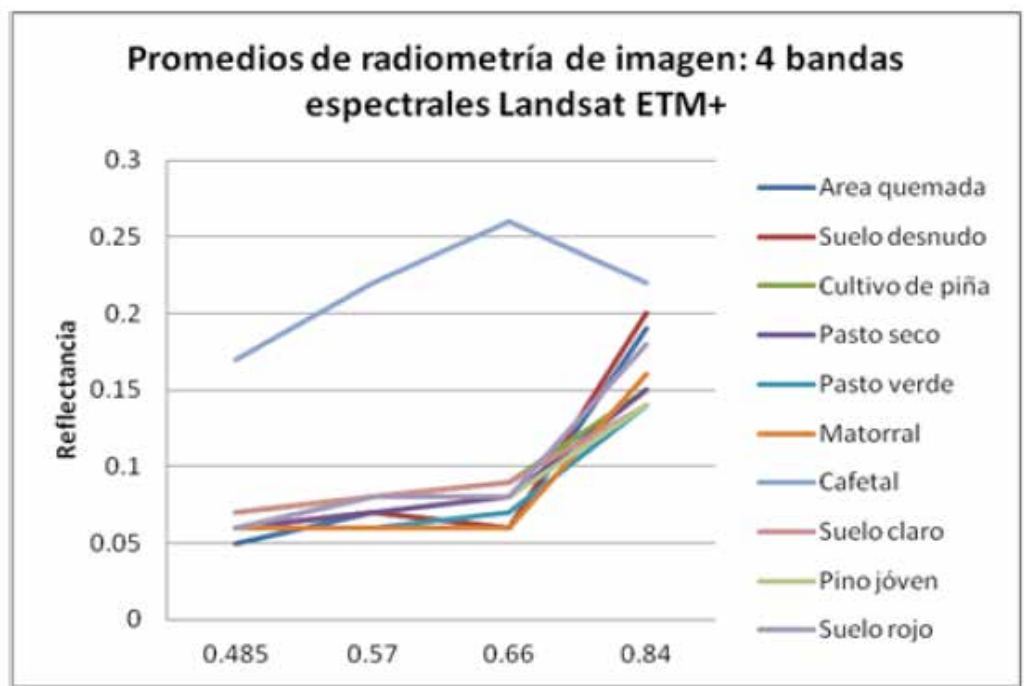

Figura 5. Gráfico de radiometría de imagen para las 4 bandas del sensor LandSat ETM+: rango del Visible y el Infrarrojo cercano.

Los resultados de radiometría de campo se presentan en forma de Promedios, debido a que el radiómetro registra la reflectancia en más de 700 canales (bandas), por lo que se calculó el promedio entre los rangos correspondientes a las cuatro bandas de radiometría orbital (cuadros 5). La figuras 6, presenta las firmas espectrales a partir de la radiometría de campo para las coberturas identificadas, mismas que se utilizaron para la elaboración de las Fichas Digitales.

\begin{tabular}{|l|c|c|c|c|c|c|}
\hline \multirow{2}{*}{ Coberturas } & \multicolumn{7}{|c|}{ Banda Espectral } \\
\cline { 2 - 7 } & $\mathbf{0 , 4 8 5}$ & $\mathbf{0 , 5 7}$ & $\mathbf{0 , 6 6}$ & $\mathbf{0 , 8 4}$ & $\mathbf{1 , 6 5}$ & $\mathbf{2 , 2 2}$ \\
\hline Área quemada & 0,05 & 0,07 & 0,025 & 0,06 & 0,19 & 0,18 \\
\hline Suelo desnudo & 0,06 & 0,07 & 0,06 & 0,2 & 0,2 & 0,09 \\
\hline Cultivo de piña & 0,07 & 0,08 & 0,09 & 0,15 & 0,28 & 0,17 \\
\hline Pasto Seco & 0,06 & 0,07 & 0,08 & 0,15 & 0,2 & 0,12 \\
\hline Pasto Verde & 0,06 & 0,06 & 0,07 & 0,14 & 0,24 & 0,12 \\
\hline Matorral & 0,06 & 0,06 & 0,06 & 0,16 & 0,15 & 0,08 \\
\hline Cafetal & 0,17 & 0,22 & 0,26 & 0,22 & 0,34 & 0,24 \\
\hline Suelo claro & 0,07 & 0,08 & 0,09 & 0,14 & 0,33 & 0,21 \\
\hline Pino joven & 0,06 & 0,08 & 0,08 & 0,14 & 0,31 & 0,18 \\
\hline Suelo rojo & 0,06 & 0,08 & 0,08 & 0,18 & 0,23 & 0,12 \\
\hline
\end{tabular}

Cuadro 5. Radiometría de campo de las coberturas. 


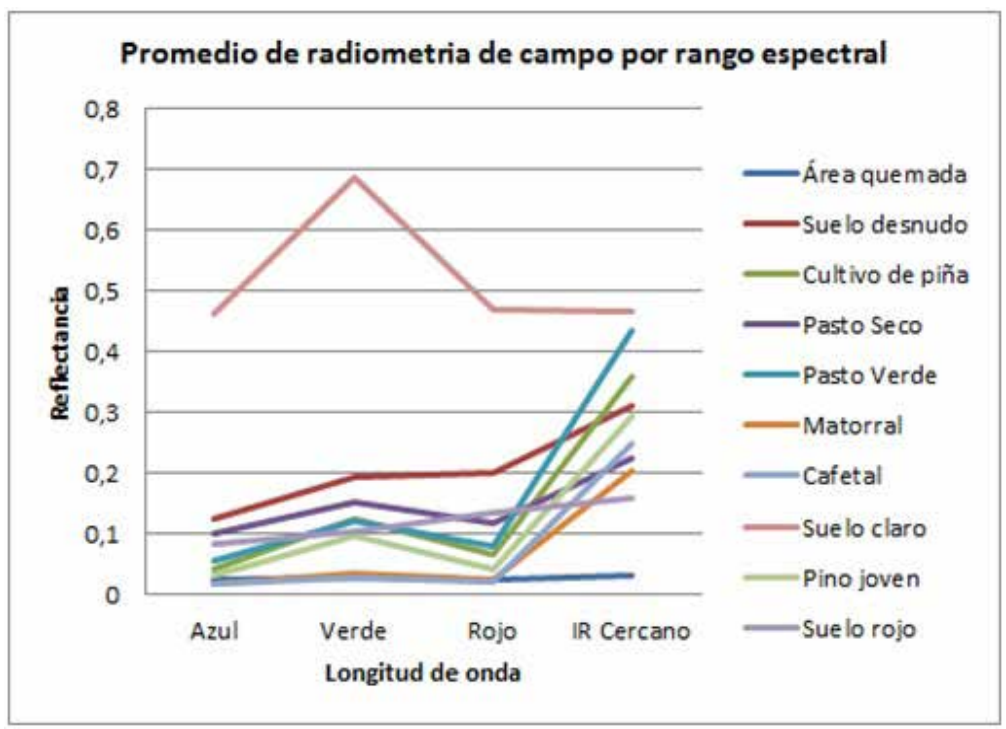

Figura 6. Gráfico de radiometría de campo.

Para las coberturas con dominancia vegetal se aplicó el Índice de Vegetación de Diferencia Normalizada: NDVI(1), por sus siglas en Inglés; para identificar la intensidad de biomasa con respecto a las condiciones fenológicas de las coberturas. Al mismo tiempo se realizó un ajuste a las condiciones del suelo: SAVI(2), por sus siglas en Inglés; para identificar el aporte que la reflectancia de fondo (reflectancia del suelo) introduce en la firma espectral de la vegetación (ver cuadro 6 , y figura 7).
(1) $\mathrm{NDVI}=(\rho \mathrm{IRC}-\rho \mathrm{R}) /(\rho \mathrm{IRC}+\rho \mathrm{R})$
(2) $\mathrm{SAVI}=\{(\rho \mathrm{IRC}-\rho \mathrm{R}) /(\rho \mathrm{RRC}+\rho \mathrm{R})+\mathrm{L}\}(1+L)$

Donde: $\quad \rho \mathrm{IRC}=$ reflectividad del infrarrojo cercano

$$
\begin{aligned}
& \rho R=\text { reflectividad del Rojo } \\
& L=\text { es una constante que depende del tipo de suelo (Domingo } \\
& \text { et all, 2008) }
\end{aligned}
$$




\begin{tabular}{|l|r|r|}
\hline \multicolumn{1}{|c|}{ Coberturas } & \multicolumn{1}{c|}{ NDVI } & \multicolumn{1}{c|}{ SAVI } \\
\hline Cultivo de piña & 0,698898718 & 0,480401442 \\
\hline Pasto Seco & 0,30545842 & 0,185665244 \\
\hline Pasto Verde & 0,690011217 & 0,523976832 \\
\hline Matorral & 0,805183501 & 0,374647295 \\
\hline Cafetal & 0,853923962 & 0,445749184 \\
\hline Pino joven & 0,760653573 & 0,455337694 \\
\hline
\end{tabular}

Cuadro 6. Calculo de los Índices NDVI y SAVI

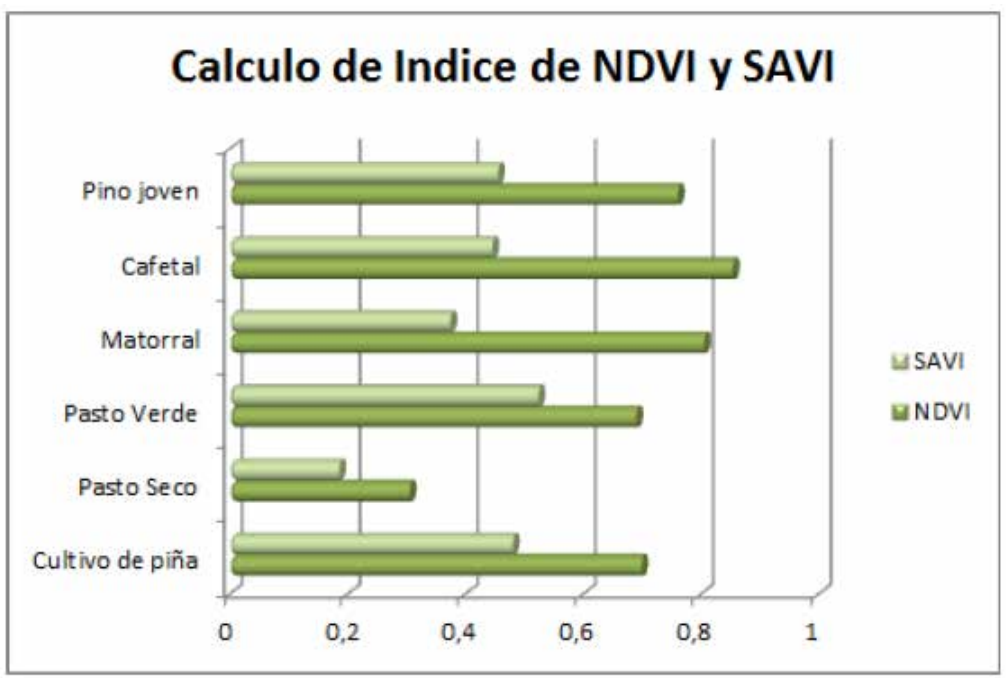

Figura 7. Gráfico de los índices de NDVI y SAVI

La correlación entre la radiometría de imagen y radiometría orbital

En el análisis de la correlación entre la radiometría de imagen (i) corregida atmosféricamente y radiometría de campo ( $r$ ), se seleccionaron solamente las dos coberturas que presentan mayor dificultad de separación espectral (cuadro 7), que son las clases Cafetal y Matorral (figuras 8 y 9 ). El análisis nos muestra que para ambas coberturas, existe alta correlación entre la radiometría de imagen y la radiometría de campo, siendo el $\mathrm{R} 2$ en ambos casos de 0.99 . Por lo tanto se afirma que se comprueba la hipótesis "Las firmas espectrales de las diferentes coberturas de la tierra, adquiridas en campo con un espectro radiómetro, son semejantes a 
las firmas espectrales orbitales de la imagen corregida por dispersión de Rayleigh, siempre que la imagen sea de la misma fecha de observación."

Cuadro 7. Correlación de las firmas espectrales de las clases: Matorral y Cafetal.

Cafetal (r)
\begin{tabular}{|c|r|r|r|}
\hline 0,01830303 & Cafetal (i) & 0,05 \\
\hline 0,02619753 & 0,07 \\
\hline 0,0194918 & 0,06 \\
\hline 0,24738017 & 0,19 \\
\cline { 2 - 3 } & 0,01595455 & 0,06 \\
\hline 0,03440741 & 0,07 \\
\hline 0,02190164 & 0,06 \\
\hline 0,20294215 & 0,2 \\
\hline
\end{tabular}

(r) radiometría de campo, (i) radiometría de imagen

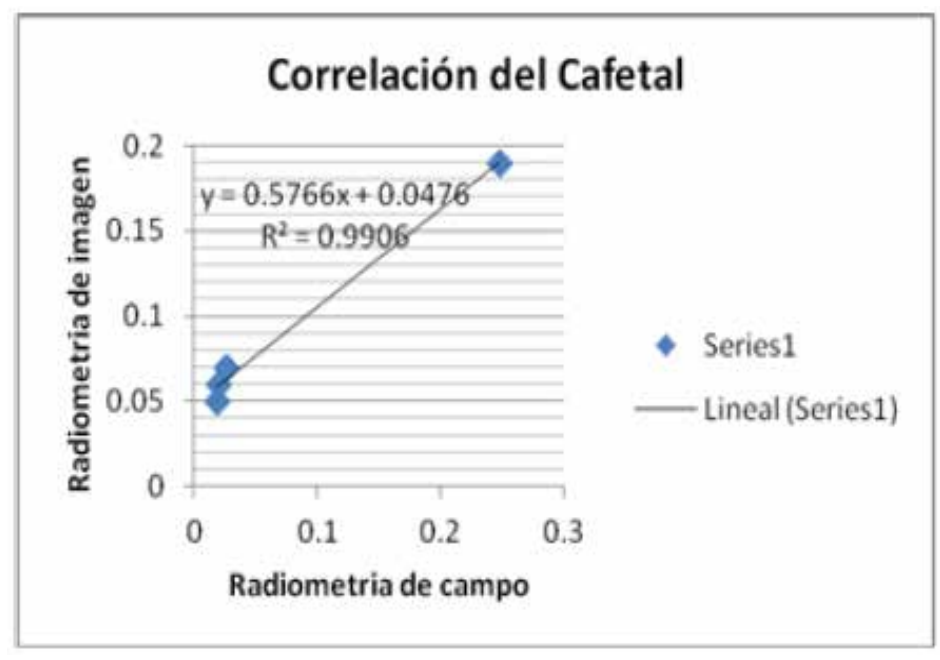

Figura 8. Correlación del cafetal 


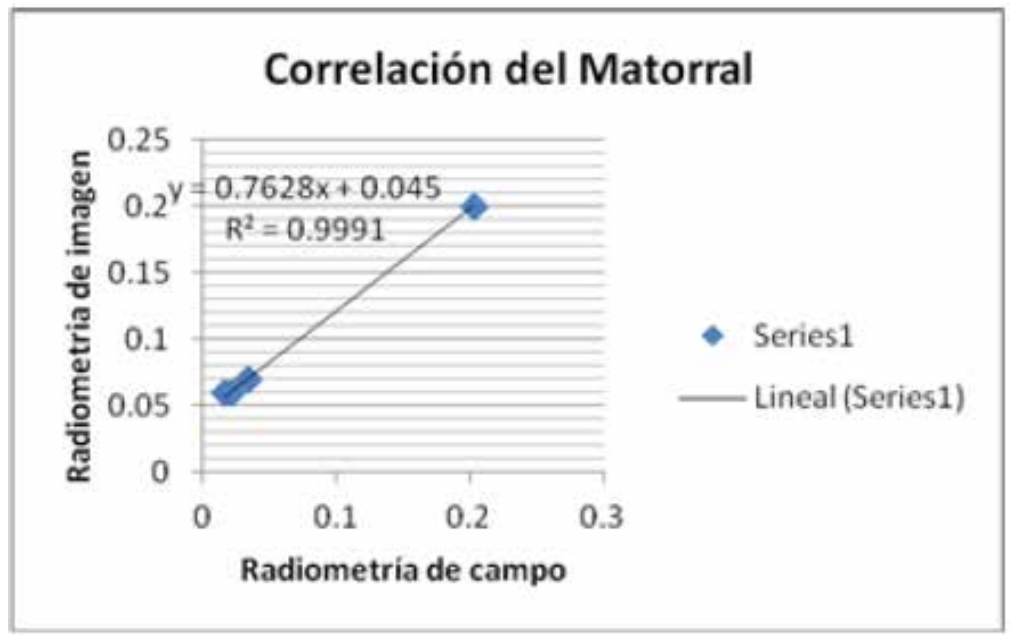

Figura 9. Correlación Matorral

La interpretación de la correlación una vez que observamos los resultados de los datos numéricos y los contrastamos con los gráficos, nos damos cuenta que presenta un caso clásico del Cuarteto de Anscombe (Anscombe, 1973), donde evidentemente muestra un valor atípico que provoca un coeficiente de correlación alto, incluso cuando la relación de las variables no es lineal, lo que supone que para futuros trabajos es necesario aumentar la muestra, para establecer si efectivamente existe o no una correlación entre las firmas espectrales de una imagen corregida, respecto a los datos directamente extraídos en campo con un radioespectrometro.

\section{Conclusiones}

Finalmente como conclusiones de la investigación se puede señalar que:

- El protocolo de radiometría de campo es válido para el registro de las firmas correspondientes para cada cobertura seleccionada, por tanto puede utilizarse en subsiguientes proyectos radiométricos.

- Las firmas espectrales sirven para calibrar imágenes sin correcciones radiométricas.

- El catálogo de firmas espectrales constituye una herramienta útil para los estudios de teledetección aplicada.

- Para obtener una mejor interpretación de las firmas espectrales de las coberturas con dominancia vegetal, es importante considerar las condiciones fenológi- 
cas de la cobertura y la contribución que la reflectancia de fondo (reflectancia del suelo) introduce en la firma espectral de la vegetación.

- Las firmas espectrales de las diferentes coberturas de la tierra, adquiridas en campo con un espectro radiómetro, son semejantes a las firmas espectrales orbitales de la imagen corregida por dispersión de Rayleigh, siempre que la imagen sea de la misma fecha de observación.

- Graficar los datos revela su estructura, así mismo, muestra cuando el análisis presenta casos patológicos como el conjunto clásico en el Cuarteto de Anscombe. Siendo preciso el análisis de cálculos como gráficas, ya que ambas salidas contribuyen al entendimiento del fenómeno estudiado.

\section{Recomendaciones}

Se recomienda extender la investigación a otras regiones del país, para continuar enriqueciendo el catálogo de firmas espectrales a nivel nacional, mismo que contribuirá al desarrollo de las investigaciones en el campo de la percepción remota (teledetección) aplicada.

Metodológicamente, se recomienda contar con un muestreo mayor al alcanzado, con ello poder realizar los ajustes necesarios en las correlaciones de las variables estudiadas.

\section{Bibliografía}

- Anscombe, F.J. 1973. "Graphs in Statistical Analysis," American Statistician, 27 (febrero de 1973), 17-21.

- Analytic Spectral Devices, Inc. 2003. FieldSpec® UV/VNIR - HandHeld Spectroradiometer - User's Guide. Boulder, Colorado, United States of America: ASD Inc.

- Cáceres, J.D. 2013. Análisis del Comportamiento Temporal de la Biomasa en Pastos del Departamentos de Olancho en el contexto de Cambio Global-Fase I. (En prensa). 
- Corrales, E. et. al. 2004. Caracterización y Análisis de Cambios en 14 Áreas Protegidas de Honduras a partir del estudio de Datos Geoespaciales. Tesis carrera de Biología. 298 pp. Corrales Andino, R. E. 2010. Caracterización de alternación hidrotermal y dinámica de cobertura del suelo mediante métodos de teledetección, en el Valle de Choluteca, Honduras. Tesis de Maestría, UNAH. Tegucigalpa. 113 pp.

- Corrales, R. 2009. Catálogo de firmas espectrales de especies florales, en la Ciudad Universitaria, Tegucigalpa. Fase I. Revista Ciencias Espaciales, No.1 Vol.1. Pp. 91-104.

- Corrales, R. 2010. Catálogo de firmas espectrales de especies florales, en la Ciudad Universitaria, Tegucigalpa. Fase II. Revista Ciencias Espaciales, No.1 Vol.3. Pp. 69-85.

- Domingo, C. et all. 2009. Detección de Pautas de Estrés Hídrico en tres Especies Forestales de Catalauña mediante Imágenes Terra-Modis. XIII Congreso Asociación Española de Teledetección, España.

- Epema, G. F. 1999. Radiometría de campo en la enseñanza de la Teledetección. Serie Geográfica, 1992, vol. 2, pp. 121-126. Departamento de Geografía, Universidad de Alcalá de Henares.

- Fernández Copel, I.A. y E. Herrero Llorente. (s/a). EL SATELITE LANDSAT. ANÁLISIS VISUAL DE IMÁGENES OBTENIDAS DEL SENSOR ETM+ SATALITE LANSADT. Departamento de Ingeniería Agrícola y Forestal, Universidad de Valladolid. Palencia, España. pp. 37.

- INTELSIG. 2003. Evaluación de la Cobertura de la Tierra en la Reserva del Hombre y la Biosfera del Rio Plátano: Análisis Multitemporal de Imágenes del Satelite Landsat-Tm, entre los aňos 2001-2002. AFE-COHDEFOR/KFW/GTZ/ GFA.

- Martín, P. y A. Sánchez. 2010. "Taller Sensores Remotos y Radiometría de Campo". I Congreso Internacional de Ordenamiento Territorial y Tecnologías de la Información Geográfica en: faces.unah.edu.hn/ctig/sitios/congreso/programdetal.html 4 de marzo de 2013.

- Ochoa, L. 2005. Detección de Cambios en el Parque Nacional la Tigra. Departamento de Francisco Morazán, Honduras Centroamérica. Proyecto de Fin de Máster, Universidad de Alcalá de Henares. 80 pp. 
- $\quad$ Pineda de Carías, M.C.; V. L. Ochoa y R. E. Corrales. 2012. Detección de cambios en la cobertura de tierra de un sector del occidente de Honduras Periodo 1991-2006. Revista Ciencia y Tecnología, número 11, pp. 78-109.

- Rodríguez Carías, C.I. 2014. Aplicación de Tecnologías de Información Geográfica "Teledetección y SIG" para el Estudio Arqueológico del Corredor Mesoamericano en el Sur de Honduras. Tesis de Maestría, UNAH. Tegucigalpa. $248 \mathrm{pp}$.

- Sánchez Ramos, A. A. 2009. Análisis Multitemporal de la cobertura de la tierra en la cuenca del Valle de Jesús de Otoro entre los años 2000-2006 aplicando técnicas de teledetección. Tesis de Maestría, UNAH. Tegucigalpa. 147 pp. 